\title{
"Anthropologie de la santé et de la maladie", Journal des anthropologues, numéro 60, printemps 1995, AFA.
}

\section{Fatoumata Ouattara}

\section{(2) OpenEdition Journals}

Édition électronique

URL : http://journals.openedition.org/apad/1671

DOI : $10.4000 /$ apad. 1671

ISSN : 1950-6929

Éditeur

LIT Verlag

\section{Édition imprimée}

Date de publication : 1 juin 1995

\section{Référence électronique}

Fatoumata Ouattara, « "Anthropologie de la santé et de la maladie", Journal des anthropologues, numéro 60, printemps 1995, AFA. », Bulletin de l'APAD [En ligne], 9 | 1995, mis en ligne le 26 juillet 2007, consulté le 21 septembre 2020. URL : http://journals.openedition.org/apad/1671 ; DOI : https:// doi.org/10.4000/apad.1671

Ce document a été généré automatiquement le 21 septembre 2020.

Bulletin de I'APAD 


\title{
"Anthropologie de la santé et de la maladie", Journal des anthropologues, numéro 60, printemps 1995, AFA.
}

\author{
Fatoumata Ouattara
}

1 Le Journal des anthropologues de l'AFA (Association Française des Anthropologues) a consacré son 60ème numéro au thème de "l'anthropologie de la santé et de la maladie".

2 Les textes réunis sous ce thème nous mettent dans le bain de la dynamique actuelle des études dans le domaine de l'anthropologie de la maladie développé en France par les travaux de M. Augé. La maladie, la santé devraient être abordées avec une approche plus holistique. Si les données ethnographiques en elles mêmes paraissent intéressantes, elles ne suffisent pas à elles seules pour rendre compte de la réalité sociale observée.

3 L'anthropologie de la santé publique et des systèmes de soins devrait selon B. Hours, constituer un nouvel objet de l'anthropologie. Pour cela, il incite à appréhender anthropologiquement les recours et stratégies thérapeutiques et les logiques sociales dans les institutions de santé. L'anthropologie du "dé-boire" est l'approche proposée par S. Fainzang dans l'étude d'une communauté d'anciens buveurs de France "Vie Libre". L'approche de l'alcoolisme comme "mal social" pose des questions méthodologiques et épistémologiques importants à l'enquête de terrain.

D. Bonnet souligne à propos du rapport "médecins - anthropologues" que c'est dans un réel travail d'équipe par la confrontation des éléments de recherche assortis de leurs problématiques respectives que les anthropologues et les médecins pourront trouver "l'égalité des termes de l'échange".

5 L'approche de la maladie en tant qu' "entreprise sociale" ne devrait pas se limiter aux seules sociétés non-occidentales mais à "tous les types de société". L'approche du sida (parce que maladie présente dans toutes les sociétés) semble bien au cœur de cette objection. De ce fait, la mobilisation rapide et importante des chercheurs autour de l'action incitative Sciences sociales et sida (J.-P. Dozon) a de multiples raisons: l'approche du sida pose des questionnements d'ordre divers à la pratique 
ethnographique. Ainsi, à partir d'une enquête sur le sida au Congo, M.-E. Gruénais montre de façon pertinente comment l'approche de cette maladie a impliqué des interrogations d'ordre méthodologiques et épistémologiques à la recherche de terrain. Par la particularité de l'objet sida, le confort ethnographique n'est plus une garantie sur le terrain. D'autre part, la réalisation du projet de recherche mettant le chercheur en relation avec les organismes nationaux travaillant sur le sujet ne facilite pas non plus la situation d'enquête. Enfin, M. E. Gruénais s'interroge sur la neutralité du chercheur devant l'informateur à propos du sida. Toujours à propos du sida, I. Bardem et I. Gobatto présentent et analysent les représentations sociales et les comportements sexuels des femmes célibataires à Ouagadougou la capitale burkinabè. Cette étude a le mérite de rompre avec une certaine conception encore trop répandue, selon laquelle la femme africaine parce que soumise, n'aurait "... pas les moyens de gérer sa sexualité". L'originalité et la finesse de cette étude ont permis de montrer que les "femmes célibataires... ont adopté, à des degrés divers, des comportements préventifs."

6 En France, l'article de Y. Marzouk sur les rapports entre patients maghrébins et praticiens hospitaliers révèle les "collusions" entre diverses "logiques du sens commun". Les patients maghrébins minoritaires en France soutiennent des représentations populaires propres à la culture dominante au Maghreb tandis que les praticiens hospitaliers manient les représentations de la culture populaire française (sens commun). Le dialogue entre les deux parties s'établit assez souvent sur des "fausses connivences et malentendus productifs" basés sur les faits de langues et de gestion de l'espace et du corps des patients à l'hôpital.

$7 \quad$ A l'issue d'un colloque qui s'est tenu à Bamako sur le thème "Médecine traditionnelle et sida" en février 1994 et qui a rassemblé guérisseurs et médecins, F. Bourdarias et A. Le Palec nous décrivent les multiples stratégies et interactions mises en œuvre par les guérisseurs en quête de reconnaissance sociale. Elles nous montrent que vis-à-vis du sida, les enjeux des guérisseurs ne correspondent pas forcément à ceux des médecins.

C. Arditi à travers une lecture critique de la thèse de K. Wyss sur l'évaluation de l'utilisation des services de santé à N'Djaména établit les limites de cette étude qui n'intègre pas (assez) les données qualitatives relatives à l'anthropologie. Cette critique établit que les conclusions de Wyss souffrent d'une sur-exploitation des données quantitatives aux dépens des matériaux qualitatifs de l'anthropologie, même si la combinaison des approches quantitatives et qualitatives est bien prônée dès le début du travail. Et c'est en cela que ce travail a un caractère "incantatoire" à propos de la "pluridisciplinarité" et de ses "mérites".

9 Dans ce numéro, on trouvera également les orientations du programme de recherche du laboratoire d'écologie humaine et d'anthropologie de l'Université d'Aix-Marseille III (dirigé par J. Benoist) ainsi que celles de l'enseignement de l'anthropologie médicale au département d'ethnologie de l'Université de Paris X-Nanterre (C. Haxaire et A. Zempléni).

10 On y trouvera également la présentation du réseau "Sociétés d'Afrique et Sida" (faite par C. Raynaut), et de l'AMADES (faite par J. Benoist).

11 Les textes publiés dans ce numéro du Journal des Anthropologues donnent au lecteur un large aperçu des nouvelles dynamiques de recherches dans le domaine de l'anthropologie de la santé et de la maladie ainsi qu'un état des débats sur la pluridisciplinarité que les nouveaux objets d'étude suscitent. 